\title{
The Relationship Between Patient Medication Adherence and Following Preventive Medicine Recommendation
}

\author{
Michal Shani, MD, MPH, Yochai Schonmann, MD, MSc, Doron Comaneshter, PhD, \\ and Alex Lustman, MbCHB, MPH
}

Background: To study the relationship between adherence to chronic medications and adherence to preventive medicine recommendations among persons with diabetes mellitus and hypertension.

Methods: Data were collected from the Clalit database included all members of Clalit aged 50 to 74 years, diagnosed with diabetes mellitus or hypertension before 2016 and treated with at least 1 medication (statins hypotensive or antidiabetic drugs) during 2017. We analyzed all the monthly prescriptions that were filled during 2017. We determined personal adherence rates by calculating each individual's average adherence rate to all the prescribed medications. Adherence rates were stratified by quintiles. We checked whether each person included followed the Israeli recommendations for influenza vaccine, colon cancer screening, and mammography.

Results: of 268,792 persons, $81.1 \%$ had hypertension, and $59.5 \%$ had diabetes; $40.6 \%$ had diabetes and hypertension. The mean age was 63.7 years; $50.6 \%$ were men. The mean number of medications used was $2.2 \pm 1.1$. An adherence rate of $\leq 20 \%$ was found in $4.2 \%$, and $>\mathbf{8 0} \%$ in $42.5 \%$. Overall, $59.6 \%$ had received an influenza vaccine, $68.0 \%$ had undergone colon cancer screening, and $75.2 \%$ of the women had undergone mammography. Increased adherence to medications was associated with increased adherence to preventive recommendations. For persons in the study cohort, adjusted odds ratios comparing the highest to the lowest quintile of medication adherence were $1.52(1.46-1.59)$ for influenza vaccine, 1.59 (1.53-1.66) for colon cancer screen, and 1.35 (1.27-1.44) for mammography.

Discussion: A positive association was observed between adherence to chronic medications and adherence to preventive medicine among persons with hypertension and diabetes. ( $\mathrm{J}$ Am Board Fam Med 2021;34:1157-1162.)

Keywords: Israel, Medication Adherence, Preventive Medicine, Primary Health Care

\section{Introduction}

Adherence to medications is key to medical care and is commonly considered in describing patient enga-

This article was externally peer reviewed.

Submitted 15 May 2021; revised 29 July 2021; accepted 30 July 2021.

From the Department of Family Medicine Sackler Faculty of Medicine, Tel Aviv University, Tel Aviv, Israel (MS, AL); Department of Family Medicine Central District, Clalit Health Service, Rehovot, Israel (MS, AL); Department of Quality Measurements and Research, Clalit Health Services, Tel Aviv, Israel (YS, DC); Siaal Research Center for Family Medicine and Primary Care, Faculty of Health Sciences, BenGurion University of the Negev, Beer-Sheva, Israel (YS).

Funding: None.

Conflict of interests: None.

Corresponding author: Dr. Michal Shani, MD, MPH, Department of Family Medicine, Central District, 56 Chen St., Rehovot, Israel, Phone: 972-8-9466394 (E-mail: michal. shani@gmail.com). gement in disease management and follow-up. Poor medication adherence has been associated with adverse outcomes and high costs of care and is of growing concern to clinicians and health care systems. ${ }^{1}$ Good adherence has been associated with long-term decreases in health care expenditure. ${ }^{2}$ Adherence of over $80 \%$ to prescribed medications was associated with fewer hospitalizations, emergency department visits, and outpatient visits among persons with various chronic diseases. ${ }^{1}$

The Israel National Program for Quality Indicators in Community Health care was established in 2000 to maintain continuous measures of health care quality that include preventive services, screening, treatment, and the management of disease. ${ }^{3}$ All 4 health maintenance organizations (HMOs) in Israel report to the national body for the quality of health. In the years 
after introducing the quality program, the performance of mammography, colon cancer screening, and influenza vaccination increased among the Israeli population. ${ }^{4,5}$ We chose mammography, colon cancer screening since they are recommended to all the patients in this age group in Israel, and influenza vaccine is recommended to all patients in this age group with diabetes and hypertension. The ongoing data collection has created the opportunity to link between adherence to preventive recommendations and medication adherence in patients with chronic diseases.

The association between adherence to preventive medicine and medication adherence has not been examined. Establishing a link between different aspects of adherence to medical interventions will better understand health behaviors; identifying those who are less likely to adhere will facilitate more comprehensive and effective interventions focusing on the poorest adherents.

We aimed to study the relationship between adherence to preventive recommendations and adherence to chronic medications among persons with diabetes mellitus and hypertension.

\section{Methods}

\section{Setting}

This is a retrospective cohort study. The study was conducted in Clalit Health Service (CHS) in Israel and was approved by the local ethics committee of Meir Health Center. Israel has universal health coverage. Since 1995, 4 HMOs provide health care to all Israeli residents. CHS is the largest HMO in Israel, serving over $52 \%$ of the population nationwide. Medical records have been fully computerized for 2 decades to create an extensive health care database. Demographic data are updated directly from the population registry of the Israeli Ministry of Interior. Laboratory tests results are recorded automatically in patients' electronic medical files and reported directly to primary care physicians. Pharmacies operated by or affiliated with CHS record all medication claims electronically and are linked to 1 central repository. CHS issues medications and requires nominal copayments of 5 to 15 USD per medication, maximal copayments for chronic medications are capped at 75 USD a month. Members buy chronic medications for up to 3 months at a time (documented as 3 separate purchases on the same date).

CHS operates a register of chronic diseases by integrating information from patient files, hospital discharges, medication claims, and laboratory results. ${ }^{6}$

\section{Study Population}

All CHS members aged 50 to 74 years were included with a documented diagnosis of diabetes mellitus or hypertension before January 1, 2016, who were treated with at least 1 relevant medication (ie, statins, hypotensive or antidiabetic drugs) during 2017. To ensure medication use throughout 2017, we included only those who filled at least 1 prescription per year in the 3 consecutive years, 2016 to 2018 , for at least 1 of the study medications. This approach was used to ensure for each medication usage before and after 2017 and to exclude patients who stopped the treatment for any reason during the study period (Dosage changes were not considered as stopping treatment). This approach was used to ensure that medications were prescribed through the whole of 2017, allowing us to calculate medication adherence more reliably.

We included 23 medications. We choose the most commonly prescribed medications for hypertension and diabetes in Israel. The hypotensive medications were: hydrochlorothiazide, 3 calcium channel blockers (nifedipine, amlodipine, and lercarnidipine hydrochloride), $3 \beta$ blockers (atenolol, metoprolol, and bisoprolol), 2 angiotensin II receptor blockers (valsartan and losartan), 3 angiotensinconverting enzyme inhibitors (ramipril, captopril, and cilazapril) and 4 statins (rosuvastatin, atorvastatin, simvastatin, and pravastatin) The antidiabetic medications included: metformin, glimepiride, repaglinide, empagliflozin, dapagliflozin, sitagliptin, vildagliptin, and linagliptin.

\section{Variables}

We analyzed all monthly prescriptions that were filled between January 1, 2017, and December 31, 2017. Filled monthly prescriptions have been found to be an accurate and inexpensive proxy for actual medication use. ${ }^{7}$ We calculated the average adherence rate for each individual's medication by dividing the actual fulfilled monthly prescriptions during 2017 by 12 and determined personal adherence rates by calculating the mean adherence rates of all the medications prescribed to each individual. The Clalit data warehouse captures all monthly medication claims reliably, but the accuracy of the data in the exact number of pills dispensed varies. We, therefore, assessed participant's adherence by calculating the proportion of months covered by a claimed prescription (a modification of PDC [proportion of days covered]). 
Demographic information included age, sex, and socioeconomic status (SES). Low SES was defined as being exempt from health care payments based on income, as determined by the National Insurance Institute of Israel. These individuals pay reduced copayments for chronic medications.

We also retrieved participants' body mass index (BMI) as the last available measurement before January 1, 2017, and family physician visits during 2017. We included "other relevant cardiovascular chronic diagnoses" as recorded in the CHS chronic disease register on January 1, 2017, and Charlson comorbidity score (for adjustment of comorbidity among patients). ${ }^{8}$ We included the mean LDL-cholesterol (LDL), HbAlc, creatinine, and hemoglobin levels recorded in 2017. We checked whether each person included actually followed the Israeli recommendation for influenza vaccine (during 2017), screening mammography (once every 2 years; ie, 2016 to 2017), and colon cancer screening (either occult blood test during 2017 or a colonoscopy in the past 10 years [2008 to 2017]). There are no copayments for influenza vaccine, mammography, or colon cancer screen.

\section{Statistical Analysis}

We calculated overall adherence rates for each medication separately. We determined personal adherence rates by calculating the mean adherence rate of each individual to all the prescribed medications examined. We stratified adherence rates by quintiles; the lowest quintile was used as a baseline.

We used logistic regression models to calculate odds ratios for adherence to influenza vaccine, colon cancer screening, and mammography, according to quintiles of medication adherence, and adjusted the model for age, gender, SES, Charlson score, and family physician visits. As a secondary analysis, we further analyzed the group according to the patients' chronic illnesses to learn whether the baseline disease influences adherence to preventive recommendations. STATA 8.0 statistical software (Stata Corp. College Station, TX, USA) was used for statistical analysis.

\section{Results}

A total of 268,792 persons were included in the study. Of them, 217,953 (81.1\%) had hypertension and 160,082 (59.5\%) had diabetes; 109,225 (40.6\%) had both diabetes and hypertension. The mean age was 63.7 years. Men comprised $50.6 \%$ of the cohort. The mean number of medications used was $2.2 \pm$
1.1. Demographic and clinical characteristics of the whole cohort according to baseline disease are described in Table 1 . The adherence rate was $\leq 20 \%$ for 11,331 (4.2\%), 21 to $40 \%$ for 20,911 (7.8\%), 41 to $60 \%$ for $47,525(17.7 \%), 61$ to $80 \%$ for 74,648 (27.8\%), and over $80 \%$ for 114,377 (42.5\%).

Of the 250,917 persons recommended to receive an influenza vaccine during 2017, 149,668 (59.6\%) received 1 . Of the 264,857 persons recommended to undergo colon cancer screening, 179,993 (68.0\%) did so. Of the 128,279 women recommended mammography, 96,491 (75.2\%) did so.

Table 2 presents odds ratios for undergoing the 3 preventive measures examined according to 3 nondistinct groups: individuals with hypertension, individuals with diabetes, and individuals with hypertension and diabetes. The odds ratios were significant from the second quintile of adherence, compared with the first quintile, and increased continually and similarly until the fourth quintile for the 3 preventive measures examined. The odds ratio also increased from the fourth to the fifth quintile of medication adherence for influenza vaccination. In contrast, the odds ratios for colon cancer screening and mammography were similar for the top 2 quintiles of medication adherence. For influenza vaccination and colon cancer screening, the odds ratios for the 3 groups (hypertension, diabetes, and combined hypertensiondiabetes) increased similarly with increased medication adherence. For mammography performance, the odds ratios for the diabetes group were lower than for hypertension and for the combined hypertension-diabetes groups, for each of the top 4 quintiles of medication adherence compared with the lowest quintile.

For each of the 3 preventive measures examined, the odds ratios increased with greater medication adherence, also after adjustment for age, sex, SES, Charlson score, and family physician visits (Table 3). Between adherence, group comparison was significant for all comparisons except for the fourth and fifth quintiles for mammography and colon cancer screening.

In multivariate logistic regressions of the whole cohort, increased number of family physician visits was associated with higher adherence to preventive recommendations.

\section{Discussion}

Among individuals with hypertension and diabetes mellitus treated with antihypertensive and antidiabetic 


\begin{tabular}{|c|c|c|c|c|}
\hline & $\begin{array}{c}\text { Total } \\
(\mathrm{N}=268,792)\end{array}$ & $\begin{array}{l}\text { Hypertension } \\
(\mathrm{N}=217,953)\end{array}$ & $\begin{array}{l}\text { Diabetes Mellitus } \\
\quad(\mathbf{N}=\mathbf{1 6 0 , 0 8 2})\end{array}$ & $\begin{array}{c}\text { Hypertension and } \\
\text { Diabetes Mellitus } \\
\quad(\mathbf{N}=109,225)\end{array}$ \\
\hline Age (50 to 74 years) (mean $\pm S D)$ & $63.7 \pm 6.3$ & $64.1 \pm 6.1$ & $63.5 \pm 6.3$ & $64.3 \pm 6.1$ \\
\hline Sex (\% women) & 49.4 & 49.7 & 48.0 & 48.0 \\
\hline Low SES (\%) & 41.2 & 40.0 & 45.8 & 45.6 \\
\hline $\mathrm{BMI}($ mean $\pm \mathrm{SD})$ & $30.0 \pm 5.5$ & $30.2 \pm 5.6$ & $30.4 \pm 5.6$ & $30.9 \pm 5.7$ \\
\hline Charlson score (mean \pm SD) & $1.9 \pm 1.7$ & $1.9 \pm 1.8$ & $2.5 \pm 1.8$ & $2.7 \pm 1.9$ \\
\hline MI (\%) & 12.2 & 13.3 & 13.6 & 16.3 \\
\hline s/p CVA (\%) & 8.8 & 10.0 & 9.7 & 12.4 \\
\hline Hemoglobin $($ mean \pm SD) & $13.6 \pm 1.4$ & $13.5 \pm 1.4$ & $13.4 \pm 1.4$ & $13.3 \pm 1.5$ \\
\hline Creatinine (mean \pm SD) & $0.9 \pm 0.5$ & $0.9 \pm 0.6$ & $0.9 \pm 0.6$ & $0.9 \pm 0.7$ \\
\hline $\mathrm{LDL}($ mean $\pm \mathrm{SD})$ & $92.7 \pm 30.3$ & $93.0 \pm 30.5$ & $87.4 \pm 29.5$ & $85.8 \pm 29.3$ \\
\hline HbA1c (mean $\pm \mathrm{SD})$ & & & $7.3 \pm 1.4$ & $7.3 \pm 1.4$ \\
\hline Number of medications ${ }^{*}($ mean $\pm S D)$ & $2.1 \pm$ & $2.2 \pm 1.1$ & $2.3 \pm 1.2$ & $2.6 \pm 1.2$ \\
\hline Number of family physician visits in 2017 (mean \pm SD) & $13.1 \pm 8.7$ & $13.3 \pm 8.7$ & $13.8 \pm 9.1$ & $14.6 \pm 9.5$ \\
\hline
\end{tabular}

BMI, body mass index; CVA, cerebrovascular event; HbA1c, hemoglobin A1c; LDL, low-density lipoprotein; MI, myocardial infarction; SD, standard deviation; SES, socioeconomic status.

*Total medications of the medications examined.

medications, increased adherence to medications was associated with increased adherence to preventive recommendations of influenza vaccine, colon cancer screen, and mammography. Individuals whose medication adherence was over $80 \%$ were $52 \%$ more likely to have influenza vaccine, $35 \%$ more likely to perform colon cancer screening, and $35 \%$ more likely to have mammography than those with a medication adherence rate lower than $20 \%$.
Among the common barriers to medication adherence that have been described among individuals with diabetes and hypertension are forgetting to take doses, disappointment that the medication does not show an immediate improvement, and feeling burned out with having to take medicines. ${ }^{9,10}$ Other factors that were identified were intolerance to medications and medications that were taken more than twice daily. ${ }^{11}$ In previous studies, we found that adherence

Table 2. Odds Ratios for Influenza Vaccine, Colon Cancer Screening, and Mammography Adherence Rate by Baseline Disease

\begin{tabular}{|c|c|c|c|}
\hline & Hypertension & Diabetes Mellitus & Hypertension and Diabetes Mellitus \\
\hline \multicolumn{4}{|l|}{ Influenza vaccine } \\
\hline Adherence rate $21 \%$ to $40 \%$ & $1.13(1.07-1.20)$ & $1.18(1.11-1.25)$ & $1.21(1.12-1.31)$ \\
\hline Adherence rate $41 \%$ to $60 \%$ & $1.25(1.19-1.32)$ & $1.30(1.23-1.37)$ & $1.31(1.21-1.41)$ \\
\hline Adherence rate $61 \%$ to $80 \%$ & $1.54(1.46-1.62)$ & $1.56(1.48-1.64)$ & $1.56(1.46-1.68)$ \\
\hline Adherence rate $>80 \%$ & $1.74(1.65-1.82)$ & $1.80(1.71-1.90)$ & $1.81(1.70-1.95)$ \\
\hline \multicolumn{4}{|l|}{ Colon cancer screening } \\
\hline Adherence rate $21 \%$ to $40 \%$ & $1.18(1.12-1.48)$ & $1.17(1.10-1.25)$ & $1.23(1.13-1.33)$ \\
\hline Adherence rate $41 \%$ to $60 \%$ & $1.36(1.30-1.43)$ & $1.37(1.30-1.48)$ & $1.40(1.30-1.50)$ \\
\hline Adherence rate $61 \%$ to $80 \%$ & $1.61(1.53-1.69)$ & $1.61(1.52-1.70)$ & $1.70(1.58-1.83)$ \\
\hline Adherence rate $>80 \%$ & $1.62(1.54-1.69)$ & $1.65(1.57-1.74)$ & $1.76(1.64-1.89)$ \\
\hline \multicolumn{4}{|l|}{ Mammography } \\
\hline Adherence rate $21 \%$ to $40 \%$ & $1.22(1.12-1.32)$ & $1.15(1.05-1.27)$ & $1.28(1.13-1.46)$ \\
\hline Adherence rate $41 \%$ to $60 \%$ & $1.29(1.20-1.39)$ & $1.19(1.09-1.30)$ & $1.30(1.16-1.46)$ \\
\hline Adherence rate $61 \%$ to $80 \%$ & $1.46(1.36-1.58)$ & $1.34(1.23-1.46)$ & $1.50(1.34-1.67)$ \\
\hline Adherence rate $>80 \%$ & $1.48(1.38-1.59)$ & $1.3691 .25-1.48)$ & $1.57(1.41-1.75)$ \\
\hline
\end{tabular}


Table 3. Odds Ratios and Adjusted Odds Ratios* for Influenza Vaccine, Colon Cancer Screening, and Mammography Adherence Rates by Medication Adherence and Patient Characteristics

\begin{tabular}{|c|c|c|}
\hline & Unadjusted Odds Ratio & Adjusted* Odds Ratio \\
\hline \multicolumn{3}{|l|}{ Influenza vaccine } \\
\hline Adherence rate $21 \%$ to $40 \%$ & $1.12(1.07-1.18)$ & $1.07(1.03-1.13)$ \\
\hline Adherence rate $41 \%$ to $60 \%$ & $1.25(1.20-1.31)$ & $1.15(1.10-1.21)$ \\
\hline Adherence rate $61 \%$ to $80 \%$ & $1.52(1.46-1.58)$ & $1.35(1.30-1.42)$ \\
\hline Adherence rate $>80 \%$ & $1.72(1.65-1.79)$ & $1.52(1.46-1.59)$ \\
\hline Age & & $1.03(1.03-1.03)$ \\
\hline Sex (women vs men) & & $0.83(0.82-0.85)$ \\
\hline Low socioeconomic status & & $1.25(1.23-1.27)$ \\
\hline Charleson score & & $1.09(1.08-1.09)$ \\
\hline Family physician visits & & $1.04(1.04-1.04)$ \\
\hline \multicolumn{3}{|l|}{ Colon cancer screening } \\
\hline Adherence rate $21 \%$ to $40 \%$ & $1.16(1.11-1.22)$ & $1.10(1.05-1.15)$ \\
\hline Adherence rate $41 \%$ to $60 \%$ & $1.36(1.30-1.42)$ & $1.21(1.16-1.26)$ \\
\hline Adherence rate $61 \%$ to $80 \%$ & $1.58(1.52-1.65)$ & $1.35(1.30-1.41)$ \\
\hline Adherence rate $>80 \%$ & $1.59(1.53-1.66)$ & $1.35(1.29-1.40)$ \\
\hline Age & & $1.03(1.03-1.03)$ \\
\hline Sex (women vs men) & & $0.98(0.97-1.00)$ \\
\hline Low socioeconomic status & & $0.99(0.97-1.00)$ \\
\hline Charlson score & & $0.96(0.95-0.96)$ \\
\hline Family physician visits & & $1.05(1.05-1.05)$ \\
\hline \multicolumn{3}{|l|}{ Mammography } \\
\hline Adherence rate $21 \%$ to $40 \%$ & $1.16(1.08-1.25)$ & $1.14(1.06-1.23)$ \\
\hline Adherence rate $41 \%$ to $60 \%$ & $1.24(1.16-1.32)$ & $1.20(1.12-1.28)$ \\
\hline Adherence rate $61 \%$ to $80 \%$ & $1.40(1.31-1.49)$ & $1.34(1.26-1.44)$ \\
\hline Adherence rate $>80 \%$ & $1.39(1.31-1.48)$ & $1.35(1.27-1.44)$ \\
\hline Age & & $1.00(0.98-1.03)$ \\
\hline Sex (women vs men) & & NA \\
\hline Low socioeconomic status & & $1.01(0.98-1.03)$ \\
\hline Charlson score & & $0.92(0.92-0.93)$ \\
\hline Family physician visits & & $1.02(1.02-1.03)$ \\
\hline
\end{tabular}

to oral medications to hypertension and diabetes varied between medications. ${ }^{12,13}$ The financial costs of the medications were not found to have an effect. This is probably due to the low costs of these medications in the Israeli national health care system.

In the current study, we showed that adherence to medication and adherence to preventive medicine increased in parallel. Notably, the adherence to mammography and colon cancer screen recommendations plateaued at a $61 \%$ to $80 \%$ medication adherence rate. Although the relationship between medication adherence and adherence to preventive medicine recommendations seems intuitive, this relationship in individuals with hypertension and diabetes mellitus has not been researched. The same factors, such as patient-physician relations, health beliefs, and risk perception, may have a similar influence on adherence to medications and the performance of preventive medicine measures. Increased adherence to influenza vaccine has been seen in patients with chronic disease, poor self-assessed health, and patients having a family doctor. ${ }^{14}$ Poor decision processes for cancer screening and medications for hypertension and hyperlipidemia were noted in a national survey in the United States. ${ }^{15}$ The relationship between adherence to preventive medicine recommendations and medication adherence may represent a healthy adherence effect $^{16}$ or may be a marker for overall healthier behavior. Further research is needed on whether investing in improving medication adherence can influence adherence to other health care activities. 


\section{Study Strengths and Limitations}

This is a data-based study. We used medication purchasing as a proxy for medication adherence, but we had no information about the actual medication use and no information about personal health beliefs, health literacy, side effects, etc. On the other hand, due to the high quality and the completeness of the databases and the information about purchasing medications before and after the study period, consistent treatment regimens and purchasing of medication over a long period may presumably indicate real use.

We had no information about various factors that might influence adherence, such as discontinuation of medications for short periods, but the large cohort and the large number of medications examined presumably decrease the effects of such phenomena.

Our study created an opportunity to evaluate overall adherence since each patient's adherence was calculated based on adherence to many commonly used medications. Moreover, most studies defined good adherence as a dichotomous measure, generally as medication adherence greater than $80 \%$. However, in everyday life, medication adherence is not a dichotomous but rather a continuous phenomenon influenced by various variables. Our study demonstrated the association between increased medication adherence and the performance of preventive medicine recommendations.

\section{Conclusion}

In a large cohort in a national health care system, an association was observed between adherence to chronic medications and adherence to preventive medicine among persons with hypertension and diabetes.

To see this article online, please go to: http://jabfm.org/content/ 34/6/1157.full.

\section{References}

1. Roebuck MC, Kaestner RJ, Dougherty JS. Impact of medication adherence on health services utilization in Medicaid. Med Care 2018;56:266-73.

2. Simon-Tuval T, Triki N, Chodick G, Greenberg D. The association between adherence to cardiovascular medications and healthcare utilization. Eur J Health Econ 2016;17:603-10.

3. The Israel National Program for Quality Indicators in Community Healthcare. Available from: https://en.israelhealthindicators.org. Accessed November 24, 2020.

4. National Program for Quality Indicators in Community Healthcare. Definition \& Results. Cancer Screening. Available from: https://en.israelhealthindicators. org/MeasuresEnglish/Cancer-Screening. Accessed November 24, 2020.

5. The Israel National Program for Quality Indicators in Community Healthcare. Elderly Health. Available from: https://en.israelhealthindicators.org/MeasuresEnglish/ 10/2. Diabetes; https://en.israelhealthindicators.org/ MeasuresEnglish/20/39. Accessed November 24, 2020.

6. Rennert G, Peterburg Y. Prevalence of selected chronic diseases in Israel. Isr Med Assoc J 2001;3:404-8.

7. Forbes CA, Deshpande S, Sorio-Vilela F, et al. A systematic literature review comparing methods for the measurement of patient persistence and adherence. Curr Med Res Opin 2018;34:1613.

8. Sharabiani MT, Aylin P, Bottle A. Systematic review of comorbidity indices for administrative data. Med Care 2012;50:1109-18. Dec.

9. Nelson LA, Wallston KA, Kripalani S, LeStourgeon LM, Williamson SE, Mayberry LS. Assessing barriers to diabetes medication adherence using the Information-Motivation-Behavioral skills model. Diabetes Res Clin Pract 2018;142:374-384.

10. Al-Noumani H, Wu JR, Barksdale D, Knafl G, AlKhasawneh E, Sherwood G. health beliefs and medication adherence in Omanis with hypertension. J Cardiovasc Nurs 2018;33:518-526.

11. Capoccia K, Odegard PS, Letassy N. Medication adherence with diabetes medication: a systematic review of the literature. Diabetes Educ 2016;42: 34-71.

12. Shani M, Lustman A, Vinker S. Adherence to oral antihypertensive medications, are all medications equal? J Clin Hypertens (Greenwich) 2019;21:243248.

13. Shani M, Lustman A, Vinker S. Diabetes medication persistence, different medications have different persistence rates. Prim Care Diabetes 2017; 11:360-364.

14. Okoli GN, Lam OLT, Racovitan F, Reddy VK, et al. Seasonal influenza vaccination in older people: A systematic review and meta-analysis of the determining factors. PLoS One 2020;15:e0234702Jun 18.

15. Fairfield KM, Gerstein BS, Levin CA, Stringfellow V, Wierman HR, McNaughton-Collins M. Decisions about medication use and cancer screening across age groups in the United States. Patient Educ Couns 2015;98:338-43.

16. Ladova K, Vlcek J, Vytrisalova M, Maly J. Healthy adherer effect - the pitfall in the interpretation of the effect of medication adherence on health outcomes. J Eval Clin Pract 2014;20:111-6. 